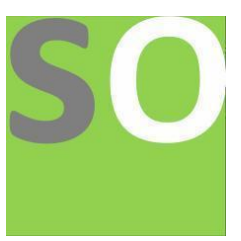

Article title: A Study on the Impact and Potential of Cryptocurrency

Authors: Aviral Srivastava[1]

Affiliations: Navrachana Higher Secondary School, Vadodara[1]

Orcid ids: 0000-0003-1164-7099[1]

Contact e-mail: meaviral17@gmail.com

License information: This work has been published open access under Creative Commons Attribution License $\mathrm{http}: / / c r e a t i v e c o m m o n s . o r g / l i c e n s e s / b y / 4.0 /$, which permits unrestricted use, distribution, and reproduction in any medium, provided the original work is properly cited. Conditions, terms of use and publishing policy can be found at https://www.scienceopen.com/.

Preprint statement: This article is a preprint and has not been peer-reviewed, under consideration and submitted to ScienceOpen Preprints for open peer review.

DOI: 10.14293/S2199-1006.1.SOR-.PP86NLN.v1

Preprint first posted online: 22 October 2021

Keywords: Cryptocurrency, Finance, Impact 


\title{
A Study on the Impact and Potential of Cryptocurrency
}

\author{
Aviral Srivastava
}

\section{ARTICLE DETAILS}

\section{Article History}

Compiled: 4 August 2021

\section{Keywords}

Cryptocurrency: It is digital money that is considered safer than real money.

Blockchain: A blockchain is a collection of blocks linked from the most recent to the Genesis block.

\section{Objectives of the study}

- To study Cryptocurrency and Blockchain.

- To analyze the impact digital currency has on the world from the environment to the financial market as well as its future potential.

- To understand the behavioral intentions with regard to usage of cryptocurrency

\begin{abstract}
Introduction: Cryptocurrency is an innovative decentralized virtual currency concept. It has emerged as a new type of investment vehicle in the world, much like gold. The current research was carried out to investigate the impact of cryptocurrency on the world market and its future potential in relation to its rising prominence among investors and traders alike.

Methods: This descriptive-analytical study was based on the interviews of individuals trading in cryptocurrencies and data collected from magazines, websites, electronic newspapers, and research articles. The collected data sets were analyzed by a data analysis model created by me.

Results: Only Cryptocurrencies with decentralized systems are trusted by investors for the fact that they can't be controlled by a single entity. Some countries have legalized crypto while some have banned them. Overall, a majority of the world still has yet to comment on the legality of Bitcoin. The cryptocurrency market is highly volatile as it is based on a simple supply and demand strategy. Bitcoin mining generates 35.95 million tons of carbon dioxide emissions and 12,000 tons of electronic waste a year. The applications of this system promise free, fast transactions, a guarantee of privacy, and a traceable supply-chain framework. Conclusion: The findings indicated that cryptocurrency has a swiftly rising user base. With its fair share of advantages and disadvantages, it is a global phenomenon. Given the correlation between investors and asset flow, its future looks promising. It is necessary to promote this next chapter in the evolution of digital assets.
\end{abstract}

\section{Research Methodology}

- This research study is based on facts and data collected from magazines, websites, electronic newspapers, and research articles.

- The secondary source of input is based on the personal views of people obtained while interviewing them. 


\section{Introduction}

Cryptocurrency is also known as a digital currency. According to Coinmarketcap, a total of 5,397 cryptocurrencies (as of April 2020) are traded on the international market. represents valuable and intangible objects that are used electronically in various applications and networks, such as online social networks, virtual payments, online games, virtual worlds, and blockchain-based peer-to-peer networks. It has the potential to alter society due to the creation of the web, changing the way we interact with family, friends, colleagues, banks, and customers, and Bitcoin, the most famous cryptocurrency that blockchain is putting on the map and that it created. great use. technology case.
Due to transparency, the tremendous growth in the blockchain user space, and the development of new businesses where cryptocurrencies are supposed to facilitate monetary activities like buying for sale, mercantilism, and of course, trading. In the world of technological advancement, cryptocurrencies are becoming increasingly convenient for investors who value privacy and money creation. The cryptocurrency is not based on the regulations of financial institutions, but is encrypted and protected, making it difficult to increase the money supply through a predefined algorithm rate. Cryptocurrencies can be converted into different types of money faster and saved in customer records.

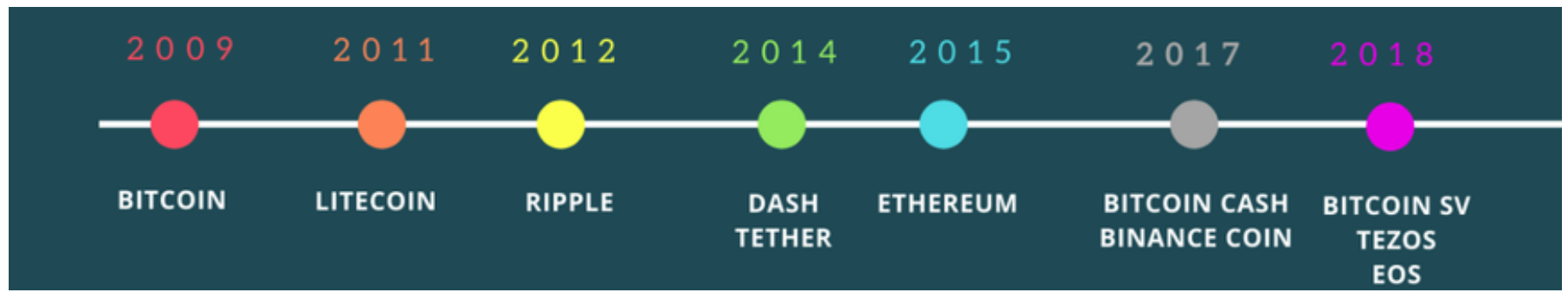

(source: bitcoinarena.net)

\section{Decentralisation}

The main idea involving all decentralized systems is that they can't be controlled by any authority. This makes them more democratic in nature, and it means that you don't need to worry about one entity being able to make off with your funds or that the system will be shut down by outside interference. There are various examples of cryptocurrencies that failed due to the sole factor that they were centralized. E.g. Libra

\section{Laws Surrounding Cryptocurrencies}

In addition to traditional banking activities, many banks are now internationally active on a large scale and are heavily involved in securities and I or insurance transactions. Their product lines change rapidly and involve very complex transactions, and have complex legal and administrative structures. In general, much of the world has still ruled on the legality of Bitcoin. Global regulators still don't fully understand the emerging industry, which could explain why some countries have yet to comment on the move. Over time, countries that have stayed on the sidelines will eventually enact a series of regulations that will either authorize the use of Bitcoin or make the activity illegal. The popularity of Bitcoin continues to exceed expectations, but not all countries will view the cryptocurrency favorably.
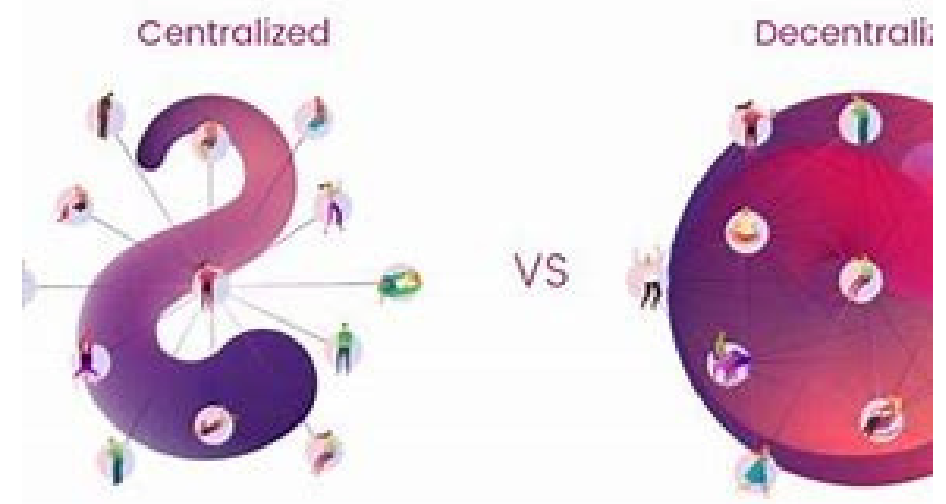


\section{Volatility}

Cryptocurrency is a very volatile market as the pricing strategy depends on supply and demand, as well as speculation.

This required approval time can vary from wallet to wallet and may even take a few days. In such cases, the investor tends to lose his chances of winning as the value of the currency fluctuates more.

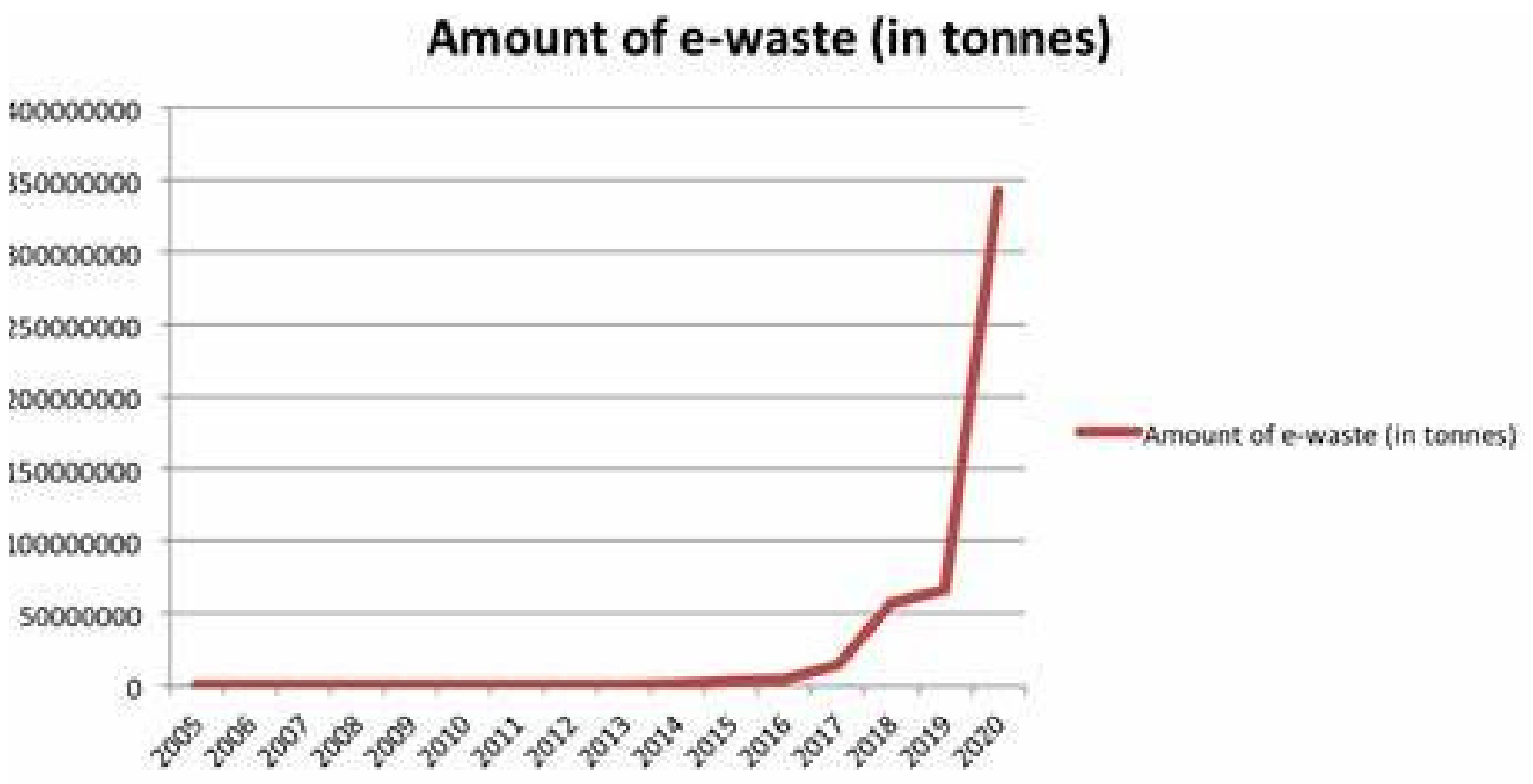

\section{Environmental Impacts}

According to researchers from the University of Cambridge, around $65 \%$ of Bitcoin mining takes place in China, a country that gets most of its electricity from burning coal. Coal and other fossil fuels are currently a major source of energy around the world, both for cryptocurrency mining and other industries. However, burning coal is a major contributor to climate change due to the resulting carbon dioxide. Bitcoin mining emits around 35.95 million tons of carbon dioxide emissions each year, roughly the same as New Zealand, according to a CNBC report.
In addition to using energy, cryptocurrency mining also generates a significant amount of e-waste because the hardware is out of date. This is especially true for application-specific ICs, specialized hardware for mining Bitcoin, Litecoin, and some other cryptocurrencies. Unlike other computer hardware, these circuits cannot be reused for other purposes and quickly become obsolete. According to Digiconomist, the Bitcoin network generates between eight and 12 thousand tons of electronic waste each year. 


\section{Internet of Things}

The Internet of Things application is an encryption technology that enables transactions between devices on the Internet of Things (IOT). IOTA addresses the transaction fees and scalability issues of blockchain technologies by ditching the block and chain. Instead, to submit a transaction to the IOTA ledger, you need to review two other previous transactions. This verification method means that there is no central ledger and miners do not need to feed the network.
Because devices on the network randomly verify each other's transactions, they create consensus on the network of connections between transactions. In crypto, this type of verification is known as a Directed Acyclical Chart (DAG), but the creators of IOTA call it a Tangle. As the computing power in Tangle grows with the network, IOTA promises fast and free transactions. It is also designed to process micropayments and payments between machines, allowing for complete machine-to-machine microeconomics.

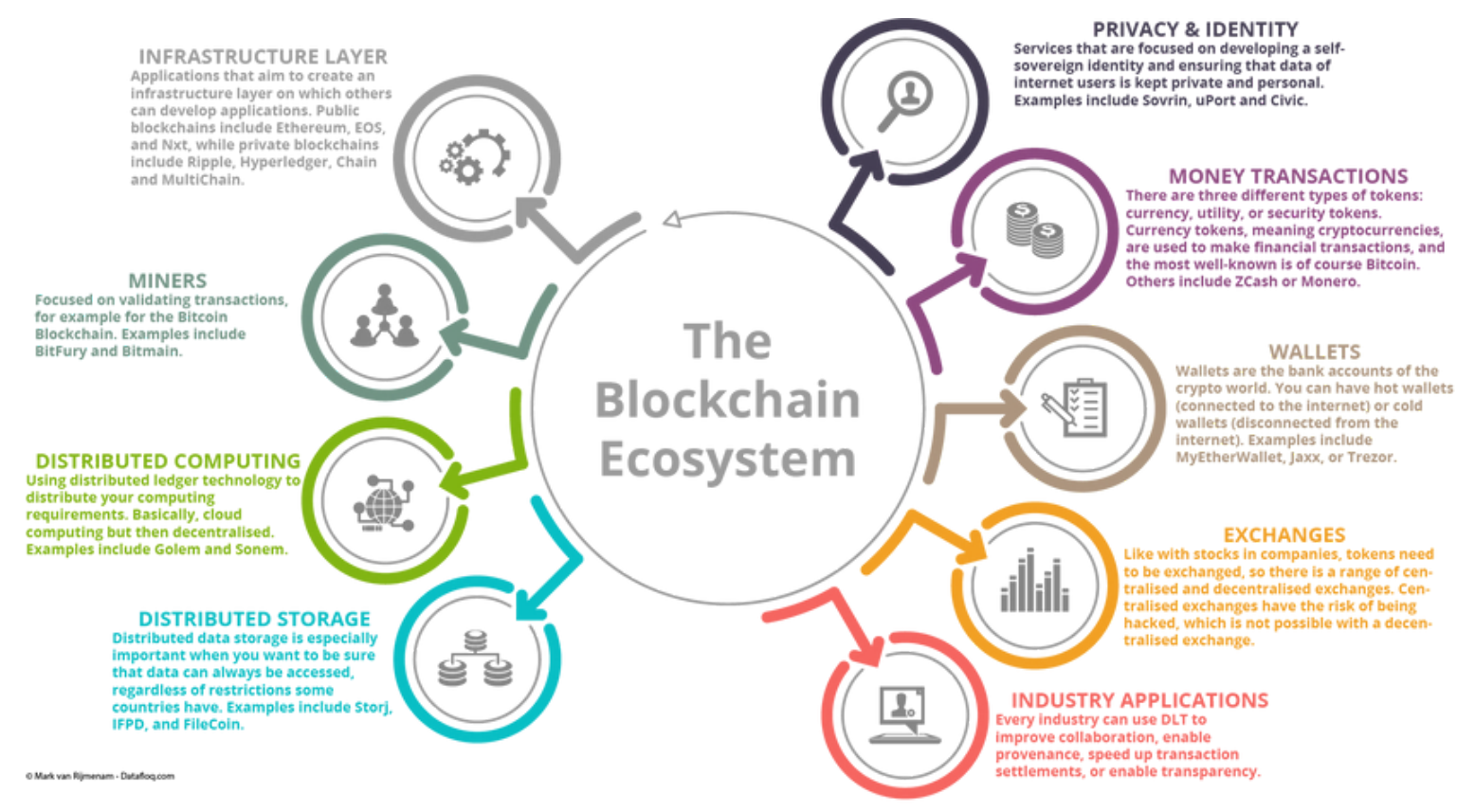

\section{Blockchain}

Blockchain is a type of database made up of a growing list of records, individually known as blocks, that are chained together using computer cryptography. The goal of the blockchain is to enable the collection and distribution of non-manipulative digital information. Unlike a typical database, in which the data is stored electronically in a "table format", the data is stored within a chain of blocks in its connected blocks, with each block containing information about the previous block. blockchains are generally managed through a network of peer-to-peer computers that work together to serve as a publicly distributed record of data (or transactions).
Each node on the network follows a specific protocol that the entire blockchain adheres to validate new blocks and communicate with each other. Although blockchain appears in almost every conversation that involves cryptocurrency, the two terms cannot be used interchangeably, but the blockchain is the network and platform through which the transaction is made and the cryptocurrency is generated. For example, the cryptocurrency Ether (ETHUSD) works through the Ethereum blockchain. 
Blockchain technology was debated in academic literature for nearly two decades before cryptocurrencies like Bitcoin (BTCUSD) and Ether, the two largest by market capitalization, became one of the first and most popular uses of blockchain. In 2009, an individual or group of people using the pseudonym "Satoshi Nakamoto" invented the first cryptocurrency on the Bitcoin blockchain, which serves as a public ledger for Bitcoin transactions.
One of the most important solutions that a digital currency offers through blockchain technology is the answer to the problem of double spending. Furthermore, it offers this full-featured solution without the need for a central authority or a trusted server as mentioned above. Since a blockchain network consists of computing nodes that can be located anywhere in the world, blockchains and therefore cryptocurrencies function as completely decentralized platforms.

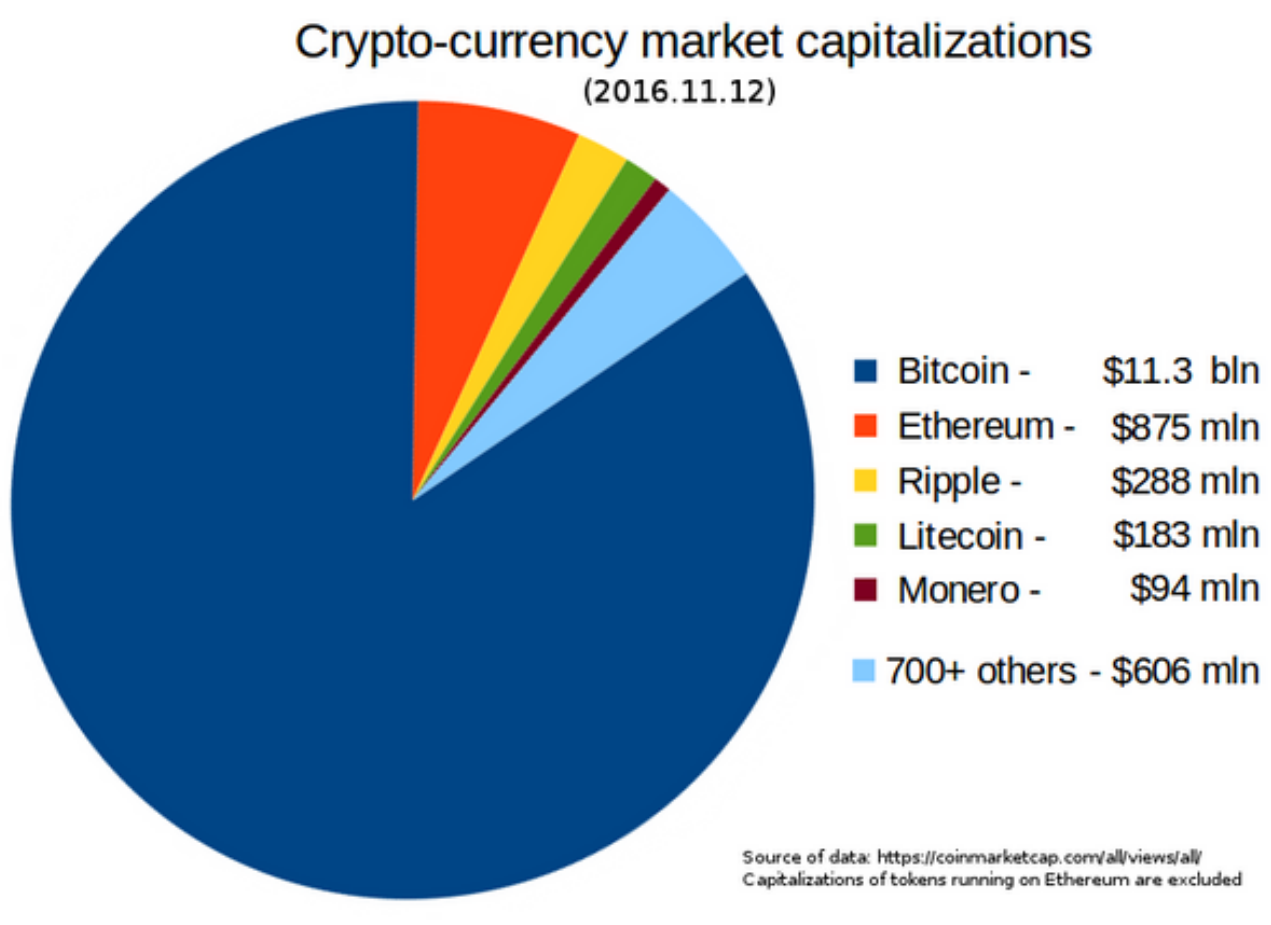

\section{Conclusion}

Cryptocurrency is one of those inventions that has become a global phenomenon. In the past, cryptocurrency has been associated with money laundering and terrorist financing. However, cryptocurrency is modern technology and a tool that we look forward to. Although there has not been a regulatory response from the government in many countries, with the exception of those where cryptocurrencies are banned, the number of investors in cryptocurrencies has grown quite rapidly in recent years. The future of cryptocurrencies looks bright and there is a ray of hope. Cryptocurrency brings huge changes in the financial sector, as well as in the individual lives of ordinary citizens.
It has its meaning, limitations, and challenges as we discussed in the information above. It's very volatile nature, the lax regulations and laws that surround its legality, and the billions of tons of garbage that are generated are its main drawbacks. However, it has its advantages in the IT field due to its decentralized nature, it would reduce cost efficiency, high transparency, and also help with trading platforms, payments, commercial financing due to blockchain integration, and the Internet of Things application that it promises free fast transactions. 


\section{References}

[1]. Mrs. Geetha R.S. (2018), a conceptual study on the impact of bitcoins on the Indian economy. International journal of business and management invention (IJBMI) ISSN: 2319-8028.

[2]. Sunidhi Kashyap. (2018), Impact of Cryptocurrency in India. IJLMH | Volume 2, Issue 1 | ISSN: 2581-5369.

[3]. https://coinmarketcap.com/

[4]. https://www.bis.org/publ/bcbs41.pdf

[5]. https://economictimes.indiatimes.com

[6]. Kurihara, Y., \& Fukushima, A. ( 2017). The Market Efficiency of Bitcoin: A Weekly Anomaly Perspective. Journal of Applied Finance \& Banking, 57-64.

[7]. Wonglimpiyarat, J. (2016). The New Darwinism of the Payment System: Will Bitcoin Replace our Cash-based Society? Journal of Internet Banking and Commerce, 1-15.

[8]. Shubhashree A. (2018). Volatility of Bitcoin and Its Implication to be a Currency. International Journal of Engineering Technology Science and Research,1017-1024

[9]. https://en.wikipedia.org/wiki/Cryptocurrency

[10]. www.coincentral.com/

[11]. www.blockgeni.com/ 